\title{
Synthesis and Characterization of a New Cellulose Acetate-Propionate Gel: Crosslinking Density Determination
}

\author{
Patrícia Allue Dantas, Vagner Roberto Botaro* \\ Departamento de Física Química, e Matemática, Universidade Federal de São Carlos, Campus Sorocaba, \\ Rodovia João Leme dos Santos, (SP-264) Km 110, Bairro do Itinga, Sorocaba, São Paulo, Brasil \\ Email: *vagner@ufscar.br
}

Received September 1, 2012; revised October 5, 2012; accepted October 16, 2012

\begin{abstract}
Crosslinking is one of the most commonly reactions used to improve the physical properties of cellulose derivatives. Cellulose Acetate Propionate (CAP) is a commercial ester obtained as a cellulose derivative and it can be used as basis for the synthesis of crosslinked chains as described in this work. Typical used crosslinkers are di-functional compounds, such as dianhydrides or diisocyanate. The formation of polymeric 3D structures as described in this work occurs typically by the reaction of the linear chains bearing free $\mathrm{OH}$ groups with crosslinking agents such as dianhydrides. These reactions are used to make a very absorbent material, typically a gel. The syntheses were performed in homogeneous medium with acetate propionate in a very dry atmosphere by employing PMDA (Pyromellitic Dianhydride) and BTDA (3,3', 4,4' Benzophenone Tetracarboxylated Dianhydride) as crosslinking agents in a reflux system. TGA analysis has shown the different thermal stability of the gels when compared with CAP. Typical TGA curves have demonstrated the lower stability of the crosslinked chains when compared to CAP as consequence of esther linkages formed in the gels structures. The Mc, which is the value for the molar mass between crosslinkings points in 3D structure, was determined according to Flory-Rehner theory. This important parameter has demonstrated greater reactivity of PMDA in comparison with the BTDA in the reactions conditions' employed in this work.
\end{abstract}

Keywords: Gel; Flory-Rehner; Crosslinking; Cellulose Acetate Propionate; TGA

\section{Introduction}

Chemical modifications of conventional organic polymers are important techniques to explore new materials having prospects of improved properties [1,2]. In many of the cases cited in the literature, the crosslinking reactions are used to improve the properties of polysaccharides, forming three-dimensional polymeric networks that swell rapidly, absorbing a large amount of water [3]. On account of this, a great demand for research, are being conducted seeking the introduction and the development of renewable polymers. In this sense, Brazil is a country with predominantly tropical climate and vast area for the cultivation of agriculture and forestry; has a great production and supply of cellulose, favoring the research in this area. Cellulose is one of the most abundant raw materials in our country, proof of this is that Brazil is the fourth biggest producer of cellulose, and which in 2010 had 2.2 million hectares of planted forests in national territory [4].

*Corresponding author.
There are several cellulose derivatives as for example esters, but only three have commercial importance: cellulose acetate (CA); a mixed Cellulose ester of Acetate and Propionate (CAP) and a mixed of cellulose acetate and butyrate (CAB) [5]. CAP is a commercial ester obtained from modification of cellulose and it can be used to synthesis of network chains, as described in this work. The formation of esters occurs when the non modified hydroxyl groups of the cellulose chains are replaced by acyl groups. Reducing the amount of $\mathrm{OH}$ groups on the cellulose chain leads to the formation of derivatives readily soluble in conventional solvents. The modification of cellulose normally forms a less crystalline material due to reduced intra and intermolecular hydrogen bonding. The nature of the substituent group and the extent, to which the hydroxyl groups are replaced, can provoke different thermal, mechanical and physical properties in the cellulose derivatives [5].

The formation of polymeric network occurs with the crosslinking of the linear chains bearing free $\mathrm{OH}$ groups and crosslinking agents as dianhydrides [6]. The proper- 
ties of a polymer network are determined by the structure and by the length of the segments between the junction points in the 3D structure of gel [7]. In the polymerizetion, the number of reactive groups of a macromolecule is growing with the increase in the number of branches, leading to the formation of complex networks. The branch of the structure can be precisely controlled and network growth is limited only by the volume of the reaction mixture [8]. The swelling balance depends on the entropy of heat of dilution, the dilation and the entropy of polymer networks. The swelling balance for a system of polymer-solvent is the density function of interweaving [9]. The swelling has a theory that was developed by Flory and Rehner in 1943, which considered that the phenomenon of swelling is basically side by three control forces: 1) The variation of entropy by mixed solventpolymer; 2) conformational entropy variation caused by the reduction in the number of conformations of chains, in consequence of their stretch and 3) Enthalpy of mixing solvent and polymer. Thus, the swelling of a given polymer is dependent on the degree of interaction between the polymer and solvent molecules, which can be referenced with the solvent/polymer interaction parameter, $\chi$ pointed out by Flory, 1943 [6].

The research on superabsorbent material began in 1960 , and continues until the present day. Many polymers have been employed in the production of gels and generally three classes are important: 1) natural [6]; 2) polyacrylamide [9] and 3) polyacrylate polymers [9-11]. The applicability of these materials depends mainly on its ability to absorb water or fluids. The ability of swelling is normally associated with the presence of hydrophilic groups like $-\mathrm{OH},-\mathrm{CONH}-,-\mathrm{COOH},-\mathrm{SO}_{3} \mathrm{H}$ and $-\mathrm{CONH}_{2}$ in its polymer network [12].

The synthesis of gels can be carried out by the same methods used for the synthesis of polymeric materials in general. Crosslinking reactions are made by introducing one or more multifunctional monomers during the growth of the polymeric chains or between two or more polymeric chains. The polymerization reaction can be initiated using radicals generated by heating, radiation ionization, by reducing agents or oxidizing [7]. The syntheses of new products with 3D polymeric structures in this work are quite new. Typically, the products are formed at room temperature its development based on the reaction of the linear chains of CAP bearing free $\mathrm{OH}$ groups with crosslinking agents as dianhydride. These reactions form of a very absorbent material, typically a gel. The syntheses were performed in homogeneous medium with acetate propionate in a very dry atmosphere by employing PMDA (Pyromellitic Dianhydride) and BTDA (3,3', 4,4' Benzophenone Tetracarboxylated Dianhydride) as crosslinking agents. One of the advantages of the process is the possibility of carrying out the reac- tion in homogeneous medium and at room temperature. In addition, the gel can be easily purified and presents a great potential for swelling in various solvents. There are great possibilities for use of gels in drug controlled release systems that are being investigated.

\section{Experimental}

\subsection{Materials}

A cellulose acetate Propionate (CAP) with average $\mathrm{Mn}$ $\sim 25,000$ with $\sim 2.5 \mathrm{wt} \%$ Acetyl, $\sim 2.6 \mathrm{wt} \%$ Hydroxyl and $\sim 45 \mathrm{wt} \%$ Propionyl, was provided by SIGMA-ALDRI$\mathrm{CH}^{\circledR}$. Pyromellitic Dianhydride (PMDA) and 3.3, 4.4 Benzophenone Tetracarboxylic Dianhydride (BTDA) with $96 \%$ and $97 \%$ of purity respectively, both provided by SIGMA-ALDRICH ${ }^{\circledR}$ were employed as crosslinkers. The solvent was acetone (propanone) with $99 \%$ of purity. The catalyst was the triethylamine with a minimum purity of $99 \%$. CAP was placed in the vacuum Oven under 625 $\mathrm{mm} \mathrm{Hg}$ vacuum, by $5 \mathrm{~h}$ at $110^{\circ} \mathrm{C}$ in order to eliminate any trace of water. The total absence of water is extremely important because crosslinking agents react quickly with water and turn into their respective acids, inhibiting the reaction with $\mathrm{OH}$ groups present in the substrate.

\subsection{Degree of Substitution (DS) Determination (ASTM D 871-96)}

$0.5 \mathrm{~g}$ of CAP samples were accurately weighted and then transferred to a $250 \mathrm{~mL}$ flask. $20 \mathrm{~mL}$ of $75 \%$ aqueous ethanol were added to each sample and then each one was heated for $30 \mathrm{~min}$ at $60^{\circ} \mathrm{C}$. Then, $20 \mathrm{~mL}$ of $0.49 \mathrm{~N}$ $\mathrm{NaOH}$ solution was added to each sample and again, heated at $60^{\circ} \mathrm{C}$ for $15 \mathrm{~min}$. The same procedure was also done for a control system (not containing CAP). The flasks were stoppered and allowed to stand at room temperature for $72 \mathrm{~h}$. The excess of alkali in the sample and in the control was titrated with $\mathrm{HCl}(0.5 \mathrm{M})$ using phenolphthalein as indicator. An excess of the acid was added $(1 \mathrm{~mL})$ and the alkali was allowed to diffuse from the regenerated cellulose overnight. The disappearance of the pink color indicated complete neutralization of the alkali. The small excess of acid was then back titrated with sodium hydroxide to a phenolphthalein end-point [7].

\subsection{Gel Synthesis}

A solution of CAP in acetone was prepared by adding c.a. 1.00 gram of the cellulose derivative in $10 \mathrm{~mL}$ of solvent under vigorous agitation. The purified anhydride was added also under agitation. The amount of modifying agent was the same as the molar quantity of free $\mathrm{OH}$ groups, considering the DS of CAP determined experimentally. Finally $1 \%$ of the catalyst in relation to CAP 
mass was added. The reaction was processed in a closed reflux system under $\mathrm{N} 2(\mathrm{~g})$ atmosphere, at $76^{\circ} \mathrm{C}$ until there was visible formation of a solid in the medium. The gel was placed in a dish and dryed in an oven at $60^{\circ} \mathrm{C}$ for 96 hours. The gels obtained with BTDA and PMDA were named CAPB and CAPP respectively. All gels were cleansed exhaustively to remove all excess of unreacted reagent and catalyst by employing a Soxhlet with acetone for 45 hours. Finally, the products were washed for 18 days in ethanol at room temperature. The gels were dried for 48 hours at $50^{\circ} \mathrm{C}$.

Scheme 1 Shows partials structures of CAPP and CAPB.

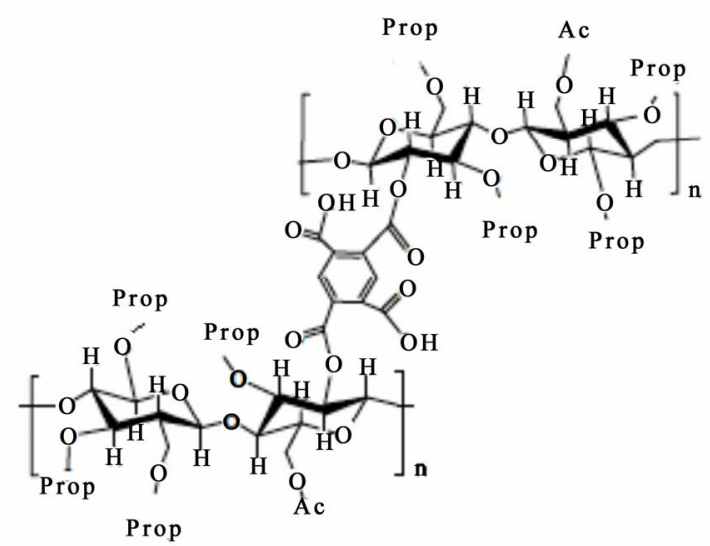

(a)

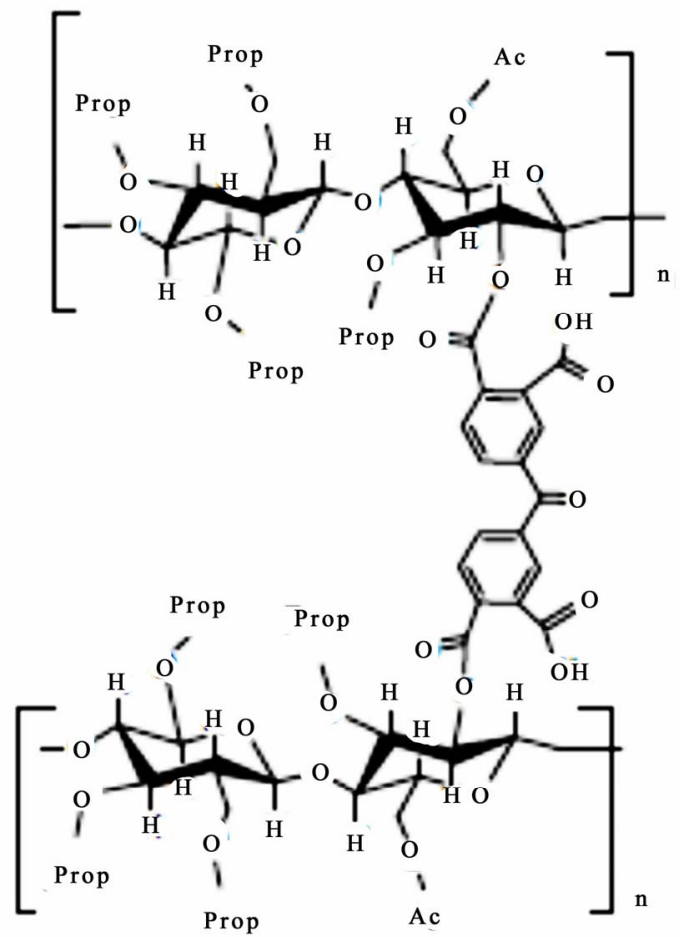

(b)

Scheme 1. Partials structures of CAPP (a) and CAPB (b).

\subsection{Polymer Characterization}

Fourier Transformed Infrared spectroscopy (FTIR) was performed by using $\mathrm{KBr}$ pellets methodology on a Nicolet IR $200^{\circledR}$. A total of 25 cumulative scans were taken with a resolution of $2 \mathrm{~cm}^{-1}$ in transmission mode. The thermo gravimetrical analyses (TGA) were carried out with in a TGA Q50-TA Instruments at heating speed of $20^{\circ} \mathrm{C} \cdot \mathrm{min}^{-1}$ at room temperature up to $800^{\circ} \mathrm{C}$, under $\mathrm{N}_{2}$ atmosphere $\left(100 \mathrm{~mL} \cdot \mathrm{min}^{-1}\right)$. SEM analysis: samples of the gels were completely dried in an oven and after treated by employing a Sputter Coater equipment of BalTec. Another details included the use of gold and Sputtering with Argon, SCD 050. The analysis was performed in the scanning electron microscope Philips XL30 model.

\subsection{Degree of Swelling in Equilibrium and Solubility Parameter of Gels}

The degree of swelling is expressed by $Q$ parameter, which is also called coefficient of swelling in balance and is determined experimentally by Equation (1)

$$
Q=\left(m_{i}-m_{o}\right) / m_{o} x \rho
$$

Were: $m_{o}=$ dry polymer mass; $m_{i}=$ polymer mass after immersion; $\rho=$ solvent density.

\subsection{Determination of Density of Crosslinks}

The crosslink density was calculated according to Equation (2):

$$
v=-[\ln (1-V r)+V r+\chi V r 2] / \rho V 1(V r 1 / 3-V r / 2)
$$

$v$ is the density of crosslink that corresponds to the number of actual chains per unit of volume. Mc and $\rho$ are the average molar mass between the crosslinkings and the density of the polymer respectively. Specimens with a mass of about $0.400 \mathrm{~g}$, cylindrical shape were cut and immersed in solvents with different solubility parameters: [ethyl ether $7.4\left(\mathrm{cal} / \mathrm{cm}^{3}\right)^{1 / 2}$ ], [ethyl acetate 9.05 (cal/ $\left.\mathrm{cm}^{3}\right)^{1 / 2}$ ], [benzene $9.2\left(\mathrm{cal} / \mathrm{cm}^{3}\right)^{1 / 2}$ ], [chloroform 9.3 $\left(\mathrm{cal} / \mathrm{cm}^{3}\right)^{1 / 2}$ ], [acetone $9.9\left(\mathrm{cal} / \mathrm{cm}^{3}\right)^{1 / 2}$ ], [ethanol 26.2 $\left(\mathrm{cal} / \mathrm{cm}^{3}\right) 1 / 2$ ] and [water $23.4\left(\mathrm{cal} / \mathrm{cm}^{3}\right)^{1 / 2}$ ]. The specimens remained submerged in $50 \mathrm{ml}$ Falcon tubes in the total absence of light to achieve equilibrium, according to ASTM471 and ASTM 1239-55 [12,13].

\section{Results and Discussion}

The determination of the DS and wt $\%$ Acetyl of CAP has confirmed the values informed by suppliers. The standard deviation for the number of three analyses was 0.11 . 


\subsection{FTIR Analysis}

New bands in gel structure gave evidence of a new material formation $[2,14]$. The typical spectrum of the CAPP shows that the product is composed of a polymer network formed with new covalent bonds and aromatic group inclusion in 3D structure. The total absence of axial deformation of $\mathrm{NH}$ salt of tertiary amine at 2680 $\mathrm{cm}^{-1}$ is an evidence of the washing process efficiency. Figure 1 shows typical FTIR spectra of CAP and CAPB gel.

Some bands are typically important in a comparative point of view; the $807 \mathrm{~cm}^{-1}$ band are assigned to angular vibration out of the plane of $\mathrm{C}-\mathrm{H}$ bonds of the aromatic ring present only at the CAPP structure. The formation of new esther linkages after crosslinkings provokes modifycation at the bands in the region of 1083 and $1740 \mathrm{~cm}^{-1}$ related to typical axial symmetric deformation of $\mathrm{C}-\mathrm{O}-\mathrm{C}$ and axial deformation of $\mathrm{C}=\mathrm{O}$ from esther respectively. Other important differences include the angular vibrations out of the plane of $\mathrm{CH}$ bonds of the ring to 753 $\mathrm{cm}^{-1}$ and the axial deformation of $\mathrm{C}-\mathrm{O}$ in the aromatic acid ester $1246 \mathrm{~cm}^{-1}$ visible in CAPP structure. Finally, another important observation is the change at the bands of $\mathrm{OH}$ groups. In CPA spectrum the absorption band occurs at $3480 \mathrm{~cm}^{-1}$, typical of aliphatic hydroxyl groups. In the gel, the $\mathrm{OH}$ groups are, to a large extent, groups of carboxylic acids formed following the crosslink of the polymer chains of CAP. In this case, absorption occurs in these hydroxyl $3440 \mathrm{~cm}^{-1}$ with typical enlargement expected for carboxylic acids.

Figure 2 shows comparative FTIR Spectra of CAPB, crosslinker and CAP.

In a general way, the same discussions presented to Figure 2 for CAPP may be considered in the discussions for the CAPB. An important band at $3485 \mathrm{~cm}^{-1}$ due to the

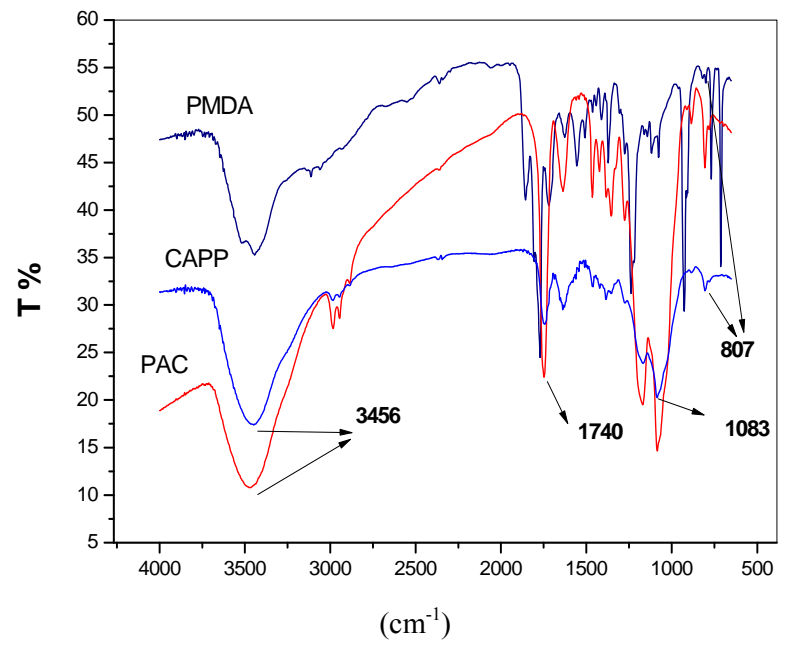

Figure 1. FT-IR spectra of PMDA, CAP and CAPP.

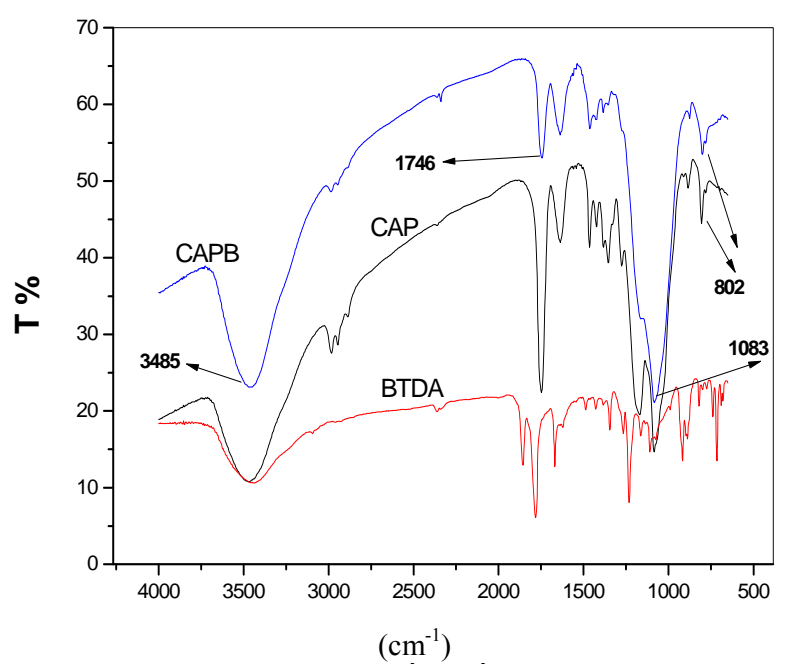

Figure 2. FT-IR spectra of BTDA, CAP and CAPB.

axial $\mathrm{OH}$ deformation is related to intermolecular hydrogen bonding which is less intense in the gel due to modification reactions. The formation of new esther linkages after crosslinkings provokes modification at the bands in the region of 1083 and $1740 \mathrm{~cm}^{-1}$ related to typical axial symmetric deformation of $\mathrm{C}-\mathrm{O}-\mathrm{C}$ and axial deformation of $\mathrm{C}=\mathrm{O}$ of esther respectively.

\subsection{TGA analysis}

Figures 3(a) and (b) clearly shows that the gels have lower thermal stability when compared to the CAP. The ester bonds formed in the gel formation process are less stable and are more easily broken by thermal degradation. This behavior has been observed for other gels obtained in our research group and published in the literature [6, 7,14].

TGA and mainly DTG curves of gels of CAPB and CAPP show two mass loss events. The first event was attributed to the degradation of the cellulose chain replacements and the second one was attributed mostly to the degradation of the polymer chain of cellulose acetate propionate. When the maximum degradation temperatures is observed, it was possible to conclude that CAPB has a degradation temperature slightly higher when compared to gel CAPP. The presence of a greater quantity of ester links in CAPP decreases the thermal stability of gels, because these links are less stable as pointed out earlier. These results are in accordance to results obtained to Mc values in Table $\mathbf{1}$.

\subsection{Solubility and Density of Crosslinkings}

The solubility parameter is an important result which characterizes the miscibility of a system under the qualitative aspect. Figures 4(a) and (b) show the solubility parameter of CAPB and CAPP respectively. 


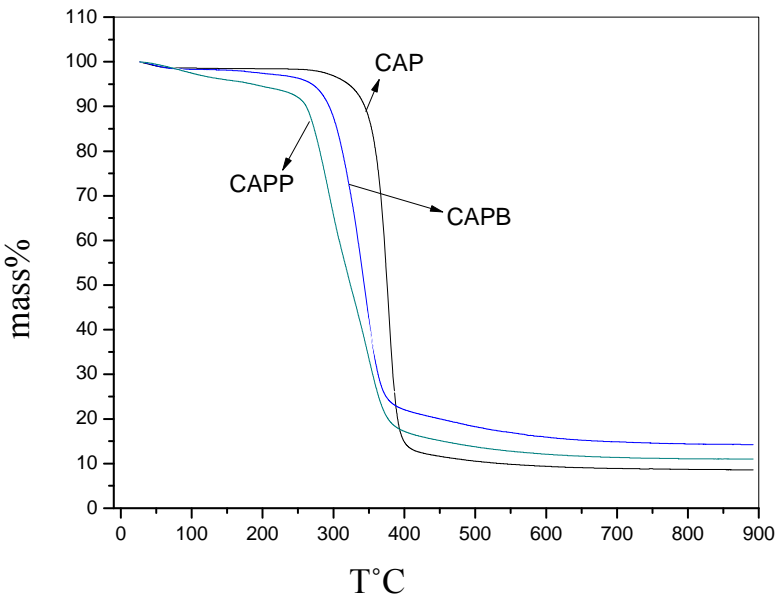

(a)

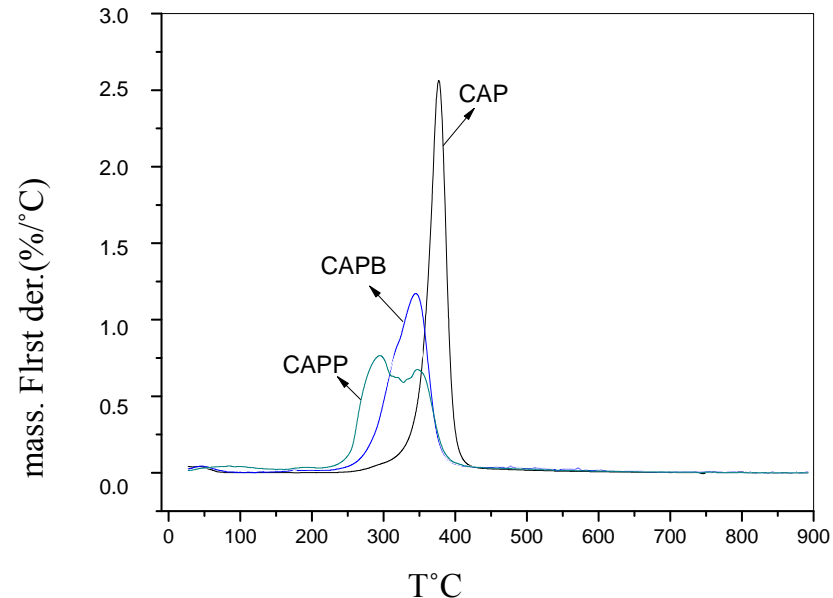

(b)

Figure 3. TGA and DTG analysis from CAP, CAPP and CAPB gels.

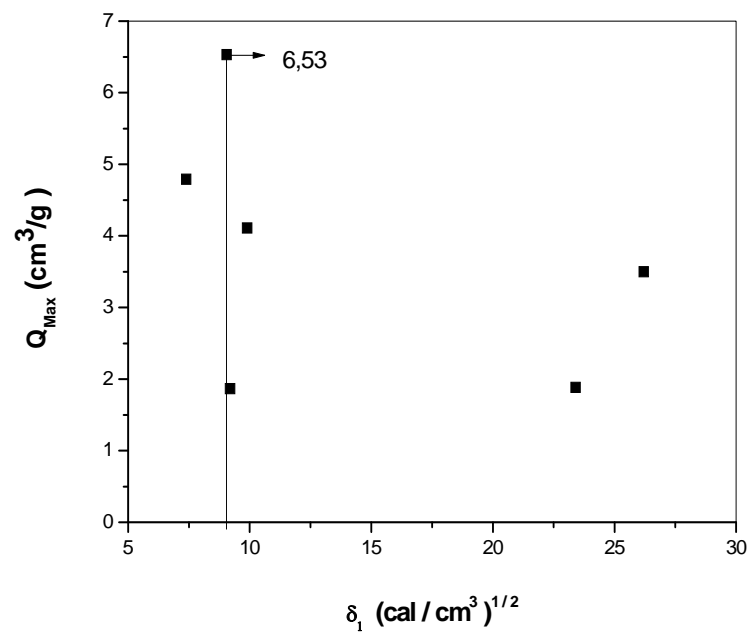

(a)

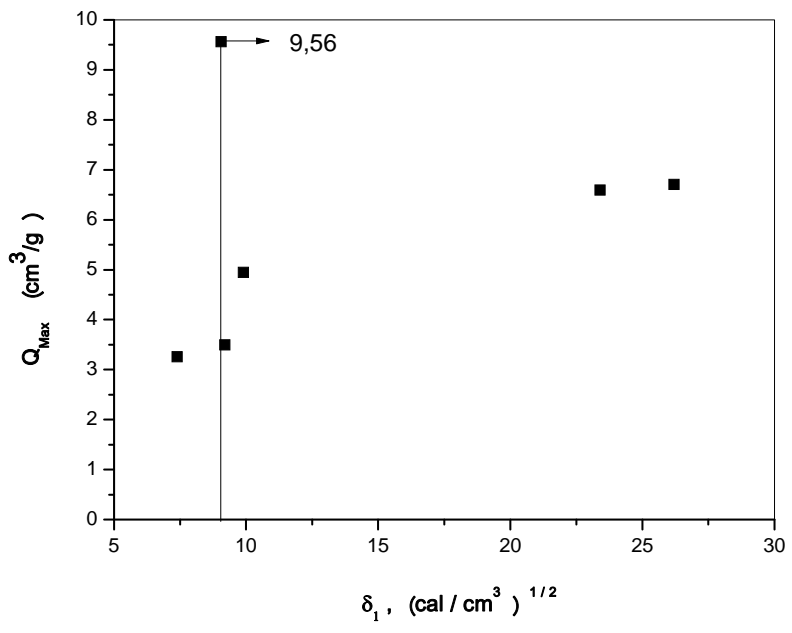

(b)

Figure 4. Solubility parameters of CAPB(a) and CAPP(b).

Table 1. Experimental data according to Flory-Rehner theory.

\begin{tabular}{ccc}
\hline Gel & CAPB & CAPP \\
\hline Percentage of absorption in the equilibrium $(\%)$ & 588 & 1062 \\
Coefficient of maximum swelling, $\mathrm{Q}_{\max }\left(\mathrm{cm}^{3} / \mathrm{g}\right)$ & 6.5 & 9.6 \\
Reduced Volume, $\mathrm{R}_{\mathrm{V}}$ & 0.2 & 0.14 \\
Density of the gel, $\rho_{2}\left(\mathrm{~g} / \mathrm{cm}^{3}\right)$ & 1.5 & 1.1 \\
Solubility parameter, $\delta^{2}\left(\mathrm{cal} / \mathrm{cm}^{3}\right)^{1 / 2}$ & 6.5 & 9.6 \\
Interaction parameter polymer-solvent, $\chi$ & 0.34 & 0.34 \\
Density of crosslinkings, $v\left(\mathrm{~g} / \mathrm{cm}^{3}\right)$ & $5.82 \times 10^{-4}$ & $2.38 \times 10^{-3}$ \\
Average molar mass between crosslinkings points, $\mathrm{Mc}\left(\mathrm{g} \cdot \mathrm{mol}^{-1}\right)$ & 2596 & 589 \\
Average numbers of repeating units between crosslinkings points & 4.8 & 1.1 \\
\hline
\end{tabular}


According to Santos [12], the polymer-solvent interacttion parameter, can be decomposed into its entropic component $(\chi s)$ and enthalpy $(\chi h)$ components according to the Equation (3)

$$
\chi=\chi h+\chi s
$$

In the maximum swelling conditions, the contribution of $\chi h$ is minimal and the interaction parameter value is practically equal to the entropic contribution. The literature has used the value of 0.34 to $\chi s$ [14]. Table 1 show the data of Flory-Rehner obtained for CAPP and CAPB gels.

The swelling theory, developed by Flory and Rehner in 1943, considerer that the phenomenon of swelling is controlled primarily by three forces: 1) the entropy variation by solvent-polymer mixture; 2) the conformational entropy variation caused by the reduction in the number of conformations of chains, in consequence of their stretch; and 3) the enthalpy of mixing solvent and polymer [5]. The swelling of a given polymer is dependent on the degree of interaction between the polymer and solvent molecules, which can be referenced with the parameter of polymer-solvent interaction. The Mc, which is the value of the molar mass between crosslinkings points in 3D structure of the gel, reveals how much the gel is crosslinked. Some important points can be considered in relation to the data presented in Table 1. The CAPB and CAPB gels have showed great power absorption and swelling in water with typical values above $500 \%$ and greater is the absorption of gel prepared with the crosslinker PMDA. The interesting power of swelling of gels is also expressed by the variable Coefficient of maximum swelling, $\mathrm{Q}_{\max }$. The Density of crosslinking and average molar mass between crosslink points show that the gel prepared with PMDA has a greater number of crosslinkings suggesting greater reactivity with this agent. A comparison between the structures of the two cross- linkers employed in the modification reactions (scheme 1) shows that the reagent PMDA is less bulky and may have less steric hindrance and can be more reactive to free hydroxyl groups present in the structure of the CAP. Finally, CAPP has both a higher density of crosslinks and a higher Qmax. The increase of crosslinkings agents increases significantly the character nonpolar of the gel. The presence of aromatic rings, typical of the structure of anhydrides, significantly affects the power of swelling of gels for the solvents employed.

\subsection{SEM Analysis}

The results of SEM show surface changes that occurred after the crosslinking reaction as indicated in Figures 5 and 6.

Observing the SEM images of CAPP and CAPB it can be observed the gels has no well-defined contours. In the same way, has no preferred geometric shape, does not have a homogeneous surface, and has several fractures in your outline and also on its surface.

Gels have also been obtained in form of films. The Figures 7 and $\mathbf{8}$ show the SEM analysis of films of CAPP and CAPB respectively.

Figures 7 and 8 show that both films have no homogeneous surfaces with internal fibrous appearance. Additionally, internal structures show extremely porous material which explains the great absorption power in solvents presented as percentage of absorption in the equilibrium (Table 1).

A comparative analysis of Figures 7 and 8 shows the gels CAPP and CAPB present some distinct characteristics. CAPP presents less gel surface homogeneity. This fact can be related with the highest degree of crosslinking and greater reactivity of PMDA as shown in Table $\mathbf{1}$. Greater crosslinking leads to a more rigid material and greater difficulty in formation of homogeneous surface after reaction.

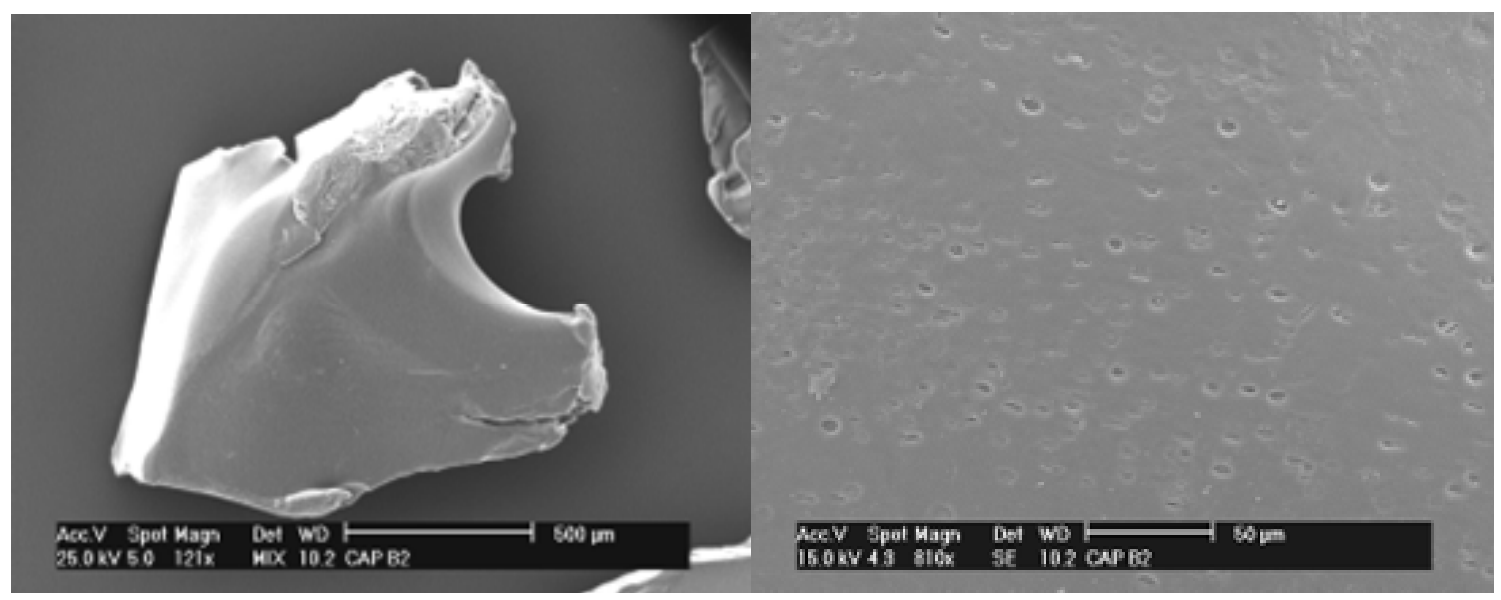

(a)

(b)

Figure 5. (a) SEM analysis showing a particle of CAPB. (b) A detail of Figure 5(a). 


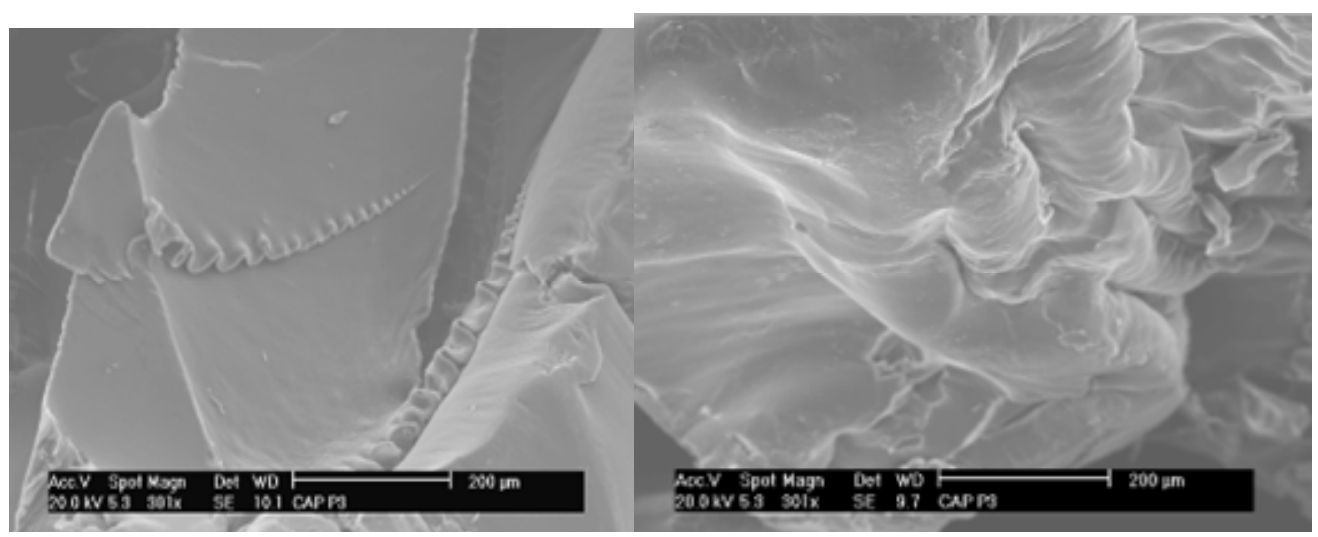

(a)

(b)

Figure 6. (a) SEM analysis showing a particle of CAPP; (b) A detail of Figure 6(a).

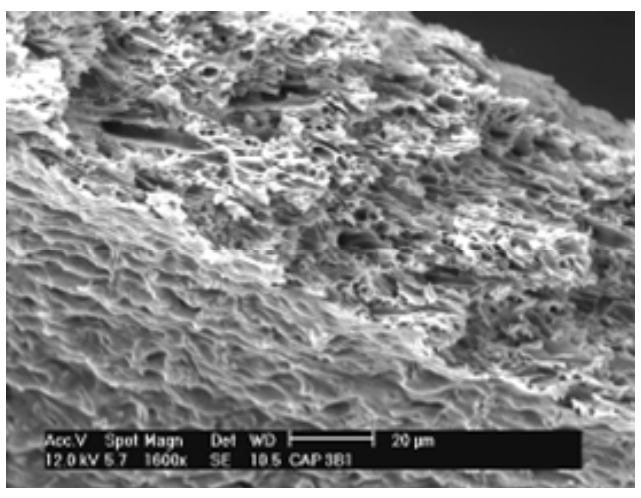

(a)

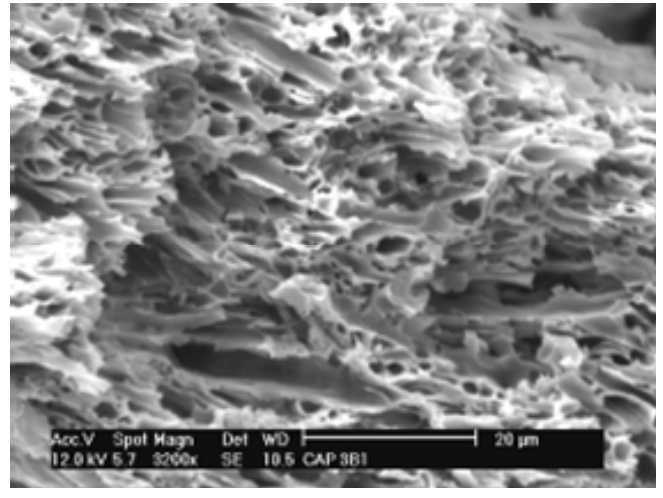

(b)

Figure 7. (a) SEM analysis showing details of CAPB film.

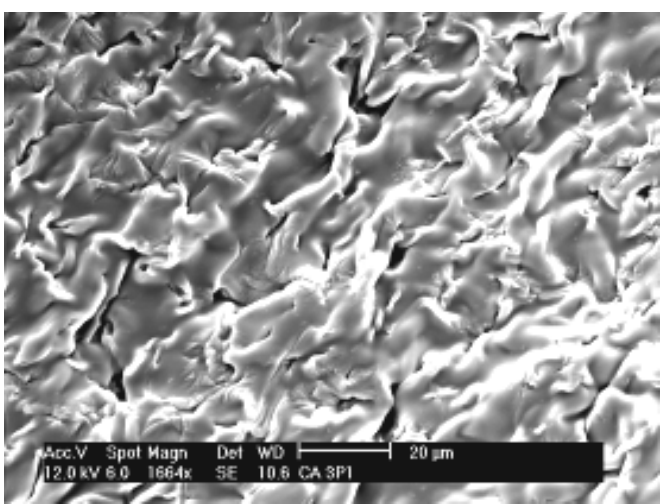

(a)

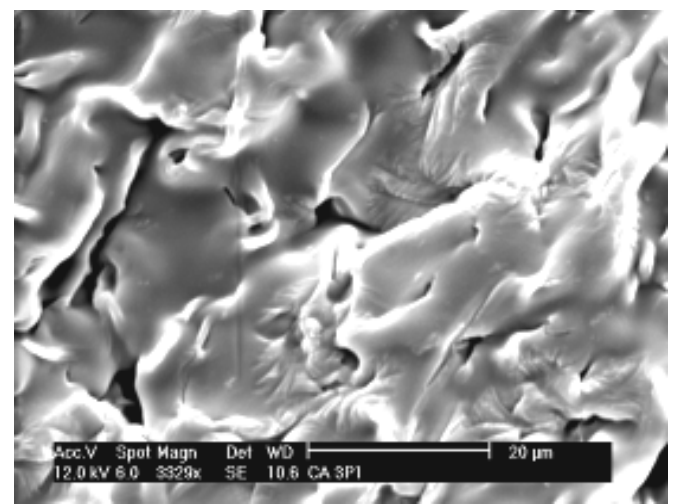

(b)

Figure 8. (a) SEM analysis showing details of CAPP film.

\section{Conclusion}

PMDA and BTDA were used as a crosslinker to crosslink a commercial esther derivative of cellulose, CAP, in this study. By changing the crosslinker agent in a homogeneous reaction, CAP gels with different degree of crosslinking density have been prepared successfully. The crosslinked products have been characterized by FTIR and TGA methods. Reduction of hydroxyl groups in FTIR spectra and the presence of typical aromatic bands were observed after modifications. The ester bonds formed in the gel formation process are less stable and are more easily broken by thermal degradation as showed by TGA analysis. The swelling theory, developed by Flory and Rehner in 1943, has successfully employed in order 
to calculate de Mc data. The Density of crosslinking and average molar mass between crosslink points show that the gel prepared with PMDA has a greater number of crosslinkings suggesting greater reactivity of this modification agent.

\section{Acknowledgements}

The authors are extremely grateful to FAPESP-project JP 2009/00855 - 0, CAPES and PPGCM-UFSCar Program.

\section{REFERENCES}

[1] M. Phiriyawirut and P. Maniaw, "Cellulose Microfibril from Banana Peels as a Nanoreinforcing Fillers for Zein Films," Open Journal of Polymer Chemistry, Vol. 2, 2012, pp. 56-62. doi:10.4236/ojpchem.2012.22007

[2] Y. Zhang, P. C. Zhu and D. Edgren, "Crosslinking Reaction of Poly(vinyl alcohol) with Glyoxal," Journal of Polymer Research, Vol. 17, No. 5, 2010, pp. 725-730. doi:10.1007/s10965-009-9362-Z

[3] S. Baljit, G. S. Chauan, S. Kumar and N. Chauan, "Synthesis, Characterization and Swelling Responses of $\mathrm{pH}$ Sensitive Psyllium and Polyacrylamide Based Hydrogels for the Use in Drug Delivery (I)," Carbohydrate Polymers, Vol. 67, No. 2, 2007, pp. 190-200. doi:10.1016/j.carbpol.2006.05.006

[4] V. R. Botaro, L. Caux and K. M. Novack, "Evaluation of Physical Properties of Bleached Eucalyptus Fibers after Thermal Treatment," Research \& Review in Polymers, Vol. 3, 2012, pp. 32-39

[5] C. S. R. Freire, A. J. D. Silvestre, C. P. Neto, R. M. A. Rocha, "An Efficient Method for Determination of the Degree of Substitution of Cellulose Esters of Long Chain Aliphatic Acids," Cellulose, Vol. 12, No. 5, 2005, pp. 449-
458. doi:10.1007/s10570-005-2203-2

[6] V. R. Botaro, V. Alvarenga and V. A. Leão, "Swelling Kinetics of Hydrogels of Cellulose," Polymeric Materials: Science \& Engineering, Vol. 23, No. 1, 2010, pp. 455 456.

[7] V. Oliveira, "Synthesis and Characterization of Cellulose Acetate Gels Crosslinkeed with Pyromelitic and 3 3' 4 4'Benzophenone Tetracarboxylic Acid Dissertação de Mestrado REDEMAT," Ouro Preto, Brasi, 2008.

[8] R. F. T. Stepto, "Polymer Networks Principles of Their Formation Structure and Properties," London, 1998

[9] P. K. Chatterjef and B. S. Gupta, "Absorbent Technology," Textile Science and Technology, Elsevier, 2002

[10] F. L. Buchholz and N. A. Peppas, "Superabsorbent Polymers, Science and Technology," ACS Symposium Series No. 573, American Chemical Society, Washington DC, 1994. doi:10.1021/bk-1994-0573

[11] C. Peniche, M. A. Cohen. B. Vazquez and J. S. Román, "Water Sorption of Flexible Networks Based on 2-Hydroxyethyl Methacrilate-Triethylenglycol Dimethacrylate Copolymers," Polymer, Vol. 38, No. 24, 1997, pp. 5977-5982. doi:10.1016/S0032-3861(96)01058-0

[12] N. Peppas, A. Nikolaos and A. R. Khare, "Preparation, Structure and Diffusional Behavior of Hydrogels in Controlled Release," Advanced Drug Delivery Reviews, Vol. 11, No. 1-2, 1993, pp. 1-35. doi:10.1016/0169-409X(93)90025-Y

[13] E. M. Santos, M. Aguiar, M. A. F. César-Oliveira, S. F Zawadzki and L. Akcelrud, "Determinação do Parâmetro de Solubilidade de Poliuretanos de PBLH," Polimeros: Ciência e Tecnologia, Vol. 10, No. 2, 2000, pp. 64-69.

[14] V. R. Botaro and V. Alvarenga, "Hidrogéis Superabsorventesa Base de Acetato de Celulose Modificado por Dianidrido BTDA," Polímeros: Cência e Tecnologia, Vol. 19, 2009 , pp. 278-284. 